\title{
The relationship of erythropoietin overexpression with von Hippel-Lindau tumour suppressor gene mutations between hypoxia-inducible factor-1 $\alpha$ and $-2 \alpha$ in sporadic clear cell renal carcinoma
}

\author{
$\mathrm{KAN} \mathrm{GONG}^{1 *}$, NING ZHANG ${ }^{2 *}, \mathrm{KAI} \mathrm{ZHANG}^{3}$ and YANQUN NA ${ }^{1}$ \\ ${ }^{1}$ Department of Urology, Peking University First Hospital; ${ }^{2}$ Department of Urology, Beijing Chaoyang Hospital; \\ ${ }^{3}$ Department of Urology, Beijing Cancer Hospital, Beijing, P.R. China
}

Received June 23, 2010; Accepted August 30, 2010

DOI: 10.3892/ijmm_00000541

\begin{abstract}
Decreased levels of von Hippel-Lindau (VHL) tumour suppressor protein are associated with up-regulation of hypoxia-inducible factor (HIF), leading to increased tumour proliferation, angiogenesis and progression. The role of erythropoietin (EPO), a target gene for HIF, remains unknown for sporadic clear cell renal cell carcinoma (sCCRCC). In this study, we determined expression levels of EPO, and its correlation with VHL mutations and HIF-1 $\alpha$ and HIF- $2 \alpha$ expression in 82 patients identified with sCCRCC following nephrectomy. We identified VHL gene alterations using multiplex polymerase chain reaction, purifying products of polymerase chain reaction, and direct sequencing. Immunohistochemical staining for HIF-1 $\alpha$, HIF- $2 \alpha$ and EPO was performed for tumour and corresponding normal tissues. Data were analyzed with respect to clinicopathological factors. EPO was detected in $87.8 \%$ of sCCRCC tumours versus $7.3 \%$ for normal tissues. EPO expression was related to tumours demonstrating VHL gene abnormalities. Of specimens with VHL alterations $95.6 \%$ tested positive for EPO, versus $78.3 \%$ when VHL gene expression was normal $(\mathrm{P}<0.01)$. EPO was identified in 96.2
\end{abstract}

Correspondence to: Dr Kan Gong, Department of Urology, Peking University First Hospital, Institute of Urology, Peking University, No. 8 Xishiku Street, Xicheng District, Beijing 100034, P.R. China E-mail: gongkan2004@yahoo.com.cn

*Contributed equally

Abbreviations: sCCRCC, sporadic clear cell renal cell carcinoma; RCC, renal cell carcinoma; VHL, von Hippel-Lindau; HIF, hypoxiainducible factor; EPO, erythroproietin; VEGF, vascular endothelial growth factor

Key words: kidney carcinoma, renal cell, erythropoietin, von HippelLindau mutation, hypoxia-inducible factor and $94.2 \%$ of HIF- $1 \alpha$ and HIF- $2 \alpha$ positive specimens, respectively, compared to 72.4 and $53.8 \%$ for HIF- $1 \alpha$ and HIF-2 $\alpha$ negative groups $(\mathrm{p}<0.01)$. Moreover, EPO expression correlated significantly with increasing nuclear grade $(\mathrm{p}<0.05)$. HIF-2 $\alpha$ was identified in $84.1 \%$ of sCCRCC, compared to $64.6 \%$ for HIF- $1 \alpha$. Expression of HIF- $1 \alpha$, HIF- $2 \alpha$ and EPO is common in sCCRCC. Although both forms of HIF up-regulate expression of EPO, the relationship to HIF-2 $\alpha$ appears to be more pronounced. The VHL-HIF-EPO pathway requires further study, as it may represent a potential molecular target for therapy of sCCRCC.

\section{Introduction}

Von Hippel-Lindau (VHL) disease is an autosomal dominant tumour syndrome with an estimated prevalence of 2-3 per 100,000 people. A germline mutation in the $V H L$ tumour suppressor gene, located on chromosome 3p25-26, predisposes carriers to tumours in multiple organs, including retinal and central nervous system hemangioblastomas, pheochromocytomas, islet cell pancreatic tumours and renal cell carcinomas (1). Advances in sporadic clear cell renal cell cancer (sCCRCC) pathobiology, attributable largely to the determination of mechanisms by which VHL exerts its tumourigenic effects, has provided the foundation for further molecular pathway research and the development of novel targeted therapies (2).

The proteolytic degradation of hypoxia-inducible factor (HIF) by the VHL tumour suppressor protein (pVHL) has emerged as a key cellular mechanism for the control of adaptive gene expression programs in response to changes in oxygen levels (1). Further, HIF transcription factors have been implicated to be a critical step in clear cell kidney tumourigenesis (3). The inactivation of pVHL mechanism is associated with up-regulation of HIF protein, inducing the activation of downstream pathways including erythropoietin (EPO), vascular endothelial growth factor (VEGF), and glycolytic pathway enzymes, thus promoting angiogenesis, proliferation and other changes in cellular metabolism that are known to promote tumour progression (4). Recent data in VHL-associated tumours such as hemangioblastomas, renal cell carcinomas and endolymphatic sac tumours implicate the 
Table I. VHL gene status, pathological stage and nuclear grade in sporadic clear cell renal cell carcinoma $(n=82)$.

\begin{tabular}{lcccccccc}
\hline \multirow{2}{*}{ VHL gene status } & \multicolumn{4}{c}{ Stage } & & \multicolumn{3}{c}{ Grade } \\
\cline { 2 - 4 } \cline { 8 - 10 } & T1 & T2 & T3 & T4 & & G1 & G2 & G3 \\
\hline Mutation & 18 & 9 & 5 & 1 & & $9(45)$ & $18(39)$ & $8(50)$ \\
Methylation & 6 & 3 & 2 & 0 & 3 & 5 & 2 \\
Normal & 26 & 7 & 5 & 0 & 8 & 23 & 6 \\
\hline
\end{tabular}

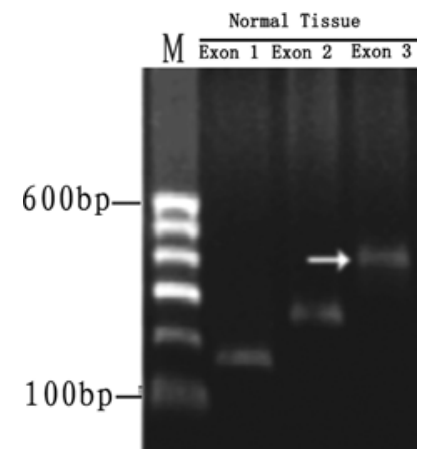

Figure 1. DNA electrophoresis of PCR primer product for VHL gene open reading frame exons $1-3$ in patient $17(\mathrm{M}, 100 \mathrm{bp}$ DNA ladder) in normal tissues. Normal and tumour tissue demonstrated three VHL-specific bands (no differences observed).

EPO expression in tumour formation via a mechanism thought to be part of the hypoxia response $(5,6)$.

The VHL tumour suppressor gene is also the most commonly mutated gene in sporadic clear cell renal cell carcinoma. This tumour subtype comprises $\sim 80 \%$ of all sporadic renal cell carcinomas, with an incidence of $\sim 80,000$ new cases annually worldwide (7). Previous studies indicate that sCCRCC shares morphologic and molecular genetic characteristics with VHL-associated RCC. The role of EPO, a proangiogenic pleiotropic cytokine that is a target for HIF, remains unknown for sCCRCC. In the present study, we determined expression levels of EPO, and its correlation with VHL mutations and HIF- $1 \alpha$ and HIF- $2 \alpha$ expression.

\section{Materials and methods}

Patients and specimens (Table I). In accordance with the principle and practices of our Institutional Review Board, this study was approved by the Ethics Committee of Peking University. From January 2000 to June 2005, 82 patients (50 males and 32 females) underwent laparoscopic or open radical nephrectomy at the Peking University First Hospital (Beijing, China) and demonstrated pathologically-confirmed sCCRCC. Surgically removed sCCRCC and counterpart normal tissues were collected from specimens and quickly frozen in liquid nitrogen. The ischemia time of renal samples was equal between laparoscopic surgery and open surgery. Each tumour underwent pathological staging based on the Union Internationale Contre le cancer/American Joint Committee on Cancer (UICC and AJCC) 2002 classification of primary RCC and Fuhrman nuclear grading: 50 (61\%), 19

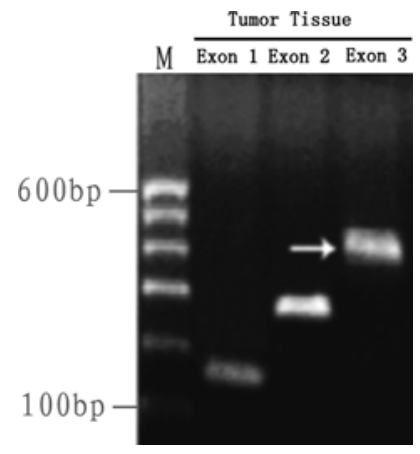

Figure 2. DNA electrophoresis of PCR primer product for VHL gene open reading frame exons $1-3$ in patient 17 (M, 100 bp DNA ladder) in tumour tissue.

$(23.2 \%), 12(14.6 \%)$ and $1(1.2 \%)$ cases were staged T1, T2, $\mathrm{T} 3$, and T4, respectively. Fuhrman grades of G1, G2, and G3 were reported for $20(24.4 \%), 46(56.1 \%)$, and $16(19.5 \%)$ cases.

Von Hipple-Lindau gene analysis. DNA was extracted from normal and tumour tissues by standard DNAzol method. Six pairs of overlapping primers were designed for the three exons in the VHL gene open reading frame as described by Gilcrease et al $(8,9)$. Polymerase chain reaction (PCR) amplification was performed using the following cycling parameters: $60 \mathrm{sec}$ at $94^{\circ} \mathrm{C}, 60 \mathrm{sec}$ at $60^{\circ} \mathrm{C}$, and $60 \mathrm{sec}$ at $72^{\circ} \mathrm{C}$ for 30 cycles (Figs. 1 and 2$)$. PCR products $(5 \mu 1)$ were analyzed by $10 \%$ PCR denaturing gradient gel electrophoresis and sent to Hua Da Zhong Sheng Biotechnology Ltd. Company, Beijing for purification and sequencing.

The methylation status of the VHL gene in the tissue samples was identified using multiplex-PCR analyses. One set of primers E1F5'-CGGAGGGCGGAGAACTGG-3' and E1 R5'-GAGGGCTCGCGCGAGTTC-3' flanked the exon 1 NotI site, and the sets E2F5'-CACCGGTGTGGCTC'IIFIAA CAA-3' and E2 R5'-ACATCAGGCAAAAATTGAGAAC TGG-3' amplified exon 2 as a control for failure of the PCR reaction. In the presence of VHL methylation, a 208-bp fragment from exon 1 was visualized, in addition to the 262-bp fragment from exon 2 (Fig. 3).

Immunohistochemical analysis of HIF-1 $\alpha, H I F-2 \alpha$ and EPO expression. Immunohistochemistry analysis was performed upon formalin-fixed, paraffin-embedded tissue sections of all specimens. Tissue sections were first soaked in xylene, and then washed in decreasing concentrations of ethanol. For 


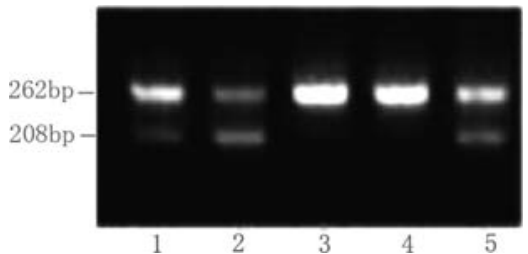

Figure 3. Multiplex-PCR analysis of VHL hypermethylation after NotI digestion. Products at $208 \mathrm{bp}$ correspond to primers flanking the NotI site, and amplify only if methylated (exon 1). Products at 262 (exon 2) confirm successful PCR. Lanes 1, 2, and 5 demonstrate VHL gene E1 hypermethylation for patients 7,49 , and 65 .

antigen retrieval, sections were treated with target retrieval solution (Dako Corporation, Carpinteria, CA) and incubated at $95^{\circ} \mathrm{C}$ for 20-30 min. Sections were cooled at room temperature, washed thrice in phosphate balanced solution and then quenched for $20 \mathrm{~min}$ in a solution containing $3 \mathrm{ml}$ $\mathrm{H}_{2} \mathrm{O}_{2}$ and $180 \mathrm{ml}$ methanol. After 3 washes in PBS, sections were drenched in $10 \%$ horse serum for $1 \mathrm{~h}$. The primary antibody was diluted in $2 \%$ horse serum, and sections were incubated in a humidified chamber at $4^{\circ} \mathrm{C}$ overnight. Primary antibodies used were HIF-1a (1:50) (OSA-602, Stressgen, Ann Arbor, MI), HIF-2a (1:50) (SC-13596, Santa Cruz Biotechnology Inc., Santa Cruz, CA), EPO (1:50) (SC-7956, Santa Cruz Biotechnology Inc.). Sections were then incubated with secondary antibody and avidin-biotin complex for $1 \mathrm{~h}$ each. Visualization was done with 3,3'-diaminobenzidine followed by counterstaining with hematoxylin. The sections were dehydrated with graded ethanol washes, xylene wash, and then mounted. All the immunohistochemistry was read by a single urological pathologist who was unaware of the different groups. The presence and intensity of antibody expression were examined in sCCRCC and normal kidney.

Positive immunohistochemistry staining for EPO was remarkable in the cytoplasm. Positive immunohistochemistry staining for HIF-1a was remarkable in cell nucleus and partial cytoplasm. Positive immunohistochemistry staining for HIF-2a was remarkable in cytoplasm. The results of immunohistochemistry were read as follows: negative (-) negative staining or positive cell $>5 \%$ per magnification $\times 200$ field; positive $\left(1^{+}\right)$positive cells $6-50 \%$ per magnification x200 field; positive $\left(2^{+}\right)$positive celsl $51-75 \%$ per magnification $\mathrm{x} 200$ field; positive $\left(3^{+}\right)$positive cells $>75 \%$ per magnification $\mathrm{x} 200$ field.

Statistical analysis. To determine correlations between the presence of VHL mutations and HIF-1 $\alpha$, HIF- $2 \alpha$, and/or EPO expression in sCCRCC, the Chi-square test was used. The Kruskal-Wallis $\mathrm{H}$ test, Fisher test, Spearman correlation analysis and logistic regression for binomial response were used for investigating the association of HIF- $1 \alpha, \mathrm{HIF}-2 \alpha$ with EPO. SPSS 11.0 (SPSS Inc., Chicago, IL) software was used for all statistical computations and results were considered statistically significant at $\mathrm{p}<0.05$.

\section{Results}

Mutational analysis of VHL coding regions. No mutations were found in normal renal tissues. VHL mutations were
Table II. The relationship between HIF-1 $\alpha$ and HIF-2 $\alpha$ expression and VHL gene status.

\begin{tabular}{lccc}
\hline & \multicolumn{3}{c}{ VHL gene status } \\
\cline { 2 - 4 } & Mutation & Methylation & Normal gene \\
\hline HIF-1 $\alpha^{+}$ & 29 & 6 & 18 \\
HIF-1 $\alpha^{-}$ & 6 & 4 & 19 \\
HIF-2 $\alpha^{+}$ & 33 & 9 & 27 \\
HIF-2 $\alpha^{-}$ & 2 & 1 & 10 \\
\hline
\end{tabular}

Table III. VHL inactivation and EPO expression in sporadic clear cell renal cell carcinoma.

\begin{tabular}{lcc}
\hline & VHL inactivation & VHL intact \\
\hline $\mathrm{EPO}^{+}$ & 43 & 29 \\
$\mathrm{EPO}^{-}$ & 2 & 8 \\
\hline
\end{tabular}

detected in 35 of $82(47.6 \%)$ sCCRCC cases. Positive DNA methylation was found in the exon 1 region of the VHL gene in 10 of 82 sCCRCC cases (12.2\%). VHL mutations occurred with similar frequency for all nuclear grades and T-stages ( $p>0.05$, Kruskal-Wallis H test).

$H I F-1 \alpha$ and HIF-2a protein expression and their correlation with VHL mutation (Table II and Fig. 4). Positive immunohistochemistry staining for HIF-1a was remarkable in cell nucleus and partial cytoplasm. Positive immunohistochemistry staining for HIF-2a was remarkable in cytoplasm. Of the 82 paired malignant and normal kidney specimens, HIF-1 $\alpha$ and HIF- $2 \alpha$ protein was undetected in all normal tissues. Conversely, HIF- $1 \alpha$ and HIF- $2 \alpha$ were detected in 53 of 82 $(64.6 \%)$ and 69 of $82(84.1 \%)$ of sCCRCC, respectively. HIF proteins were also significantly associated with VHL gene mutation; VHL gene mutations were found in 29 of the 53 $(54.7 \%)$ tumours with detectable expression of HIF-1 $\alpha$ but only 6 of 29 without detectable HIF-1 $\alpha$ protein levels (Fisher test, $\mathrm{p}<0.05)$. VHL gene mutations also occurred in 33 of the $69(47.8 \%)$ specimens with detectable expression of HIF- $2 \alpha$ compared with 2 of $13(15.3 \%$ ) without HIF-2 $\alpha$ protein (Fisher Exact test, $\mathrm{p}<0.05)$.

EPO expression (Fig. 4), VHL inactivation and HIF-lal $H I F-2 a$ expression (Tables III-V). Positive immunohistochemistry staining for EPO is remarkable in cytoplasm. EPO was detected in $72(87.8 \%)$ cases of sporadic clear cell renal cell carcinomas versus 6 cases $(7.3 \%)$ in normal tissue counterparts. The ratios of EPO expression in tumour tissue divided by corresponding normal tissue were significantly higher in tumours with known VHL inactivation (95.6\%) than in those without detectable VHL inactivation $(78.3 \%$ ) (Chi-square test, $\mathrm{P}<0.05$ ). EPO was detected in $96.2 \%$ cases expressing HIF-2 $\alpha$ and $53.8 \%$ of cases without HIF- $2 \alpha$ expression (Chi-square test, $\mathrm{P}<0.05$ ). Similar findings were observed for the relationship between EPO and HIF-1 $\alpha$, 

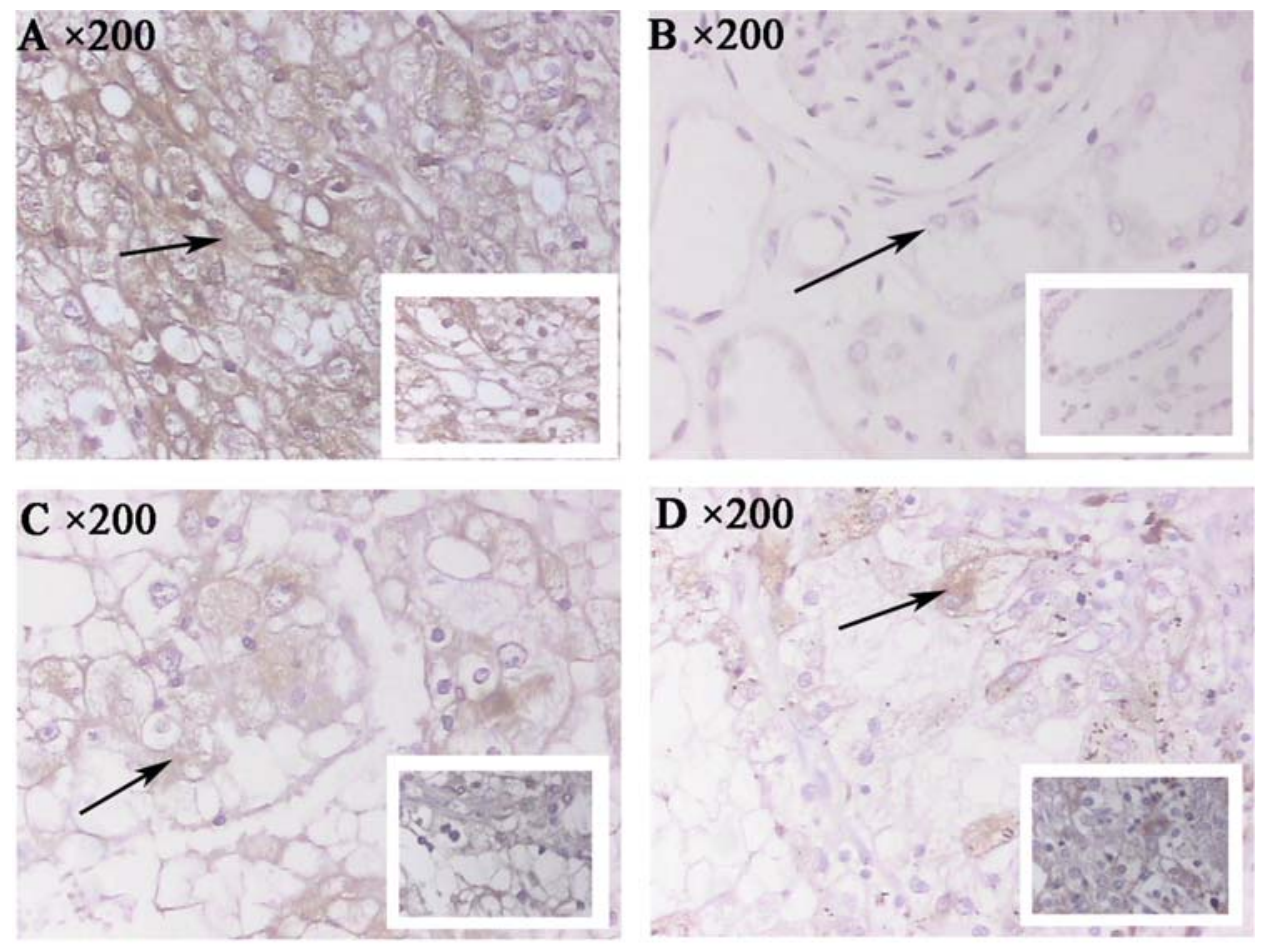

Figure 4. (A and B) Immunohistochemical staining of normal renal and clear cell renal cell carcinoma tissues of EPO. (A) Clear cell renal cell carcinoma tissue. The EPO protein was found to be well stained in the cytoplasmic area of clear cell renal cell carcinoma cells (indicated by black arrows). (B) Normal renal tissue. EPO was only weakly/absent stained in normal cells (black arrows). Inserts x400. (C and D) Immunohistochemical staining of normal renal and clear cell renal cell carcinoma tissues of HIF-1 $\alpha$ and HIF-2 $\alpha$. (C) Clear cell renal cell carcinoma tissue. The HIF-1 $\alpha$ protein was found to be well stained in the cytoplasmic area of clear cell renal cell carcinoma cells (indicated by black arrows). (D) HIC of HIF-2 $\alpha$ in clear cell renal cell carcinoma tissue. It was also well stained in the cytoplasmic area of clear cell renal cell carcinoma cells (indicated by black arrows). Inserts x400.

Table IV. Relationship between HIF-1 $\alpha$, HIF-2 $\alpha$, and EPO expression in sCCRCC.

\begin{tabular}{lcc}
\hline & $\mathrm{EPO}^{+}$ & EPO- $^{-}$ \\
\hline HIF- $1 \alpha^{+}$ & 51 & 2 \\
HIF-1 $\alpha^{-}$ & 21 & 8 \\
HIF- $2 \alpha^{+}$ & 65 & 4 \\
HIF- $2 \alpha^{-}$ & 7 & 6 \\
\hline
\end{tabular}

although HIF-2 $\alpha$ was more strongly associated with EPO expression $(\mathrm{P}<0.05$, Spearman correlation analysis and logistic regression for binomial response). No statistically significant associations between HIF- $1 \alpha$ or HIF- $2 \alpha$ expression and pathological stage or nuclear grade were observed $(\mathrm{P}>0.05$, Kruskal-Wallis $\mathrm{H}$ test), while EPO expression significantly correlated only with nuclear grade $(\mathrm{P}<0.01$, Kruskal-Wallis $\mathrm{H}$ test).

\section{Discussion}

Erythropoietin (EPO) is fully characterized as the principal hematopoietic growth factor regulating of cellular proliferation and differentiation along erythroid lineages. However, recent identification of EPO receptors in cancer cells has raised the possibility that EPO may exert direct tumourigenic effects, including stimulation of proliferation, inhibition of apoptosis, and as a key modulator for angiogenesis downstream of hypoxia-inducible factor $(10,11)$. The importance of protein expression profiles for kidney cancers has become better recognized as associations with pathobiology, prognosis, and response to treatment, as well as their potential for targetspecific molecular therapeutics $(2,12)$. Germ line VHL tumour suppressor gene loss-of-function occurs via mutation, promoter hypermethylation or large-size gene deletion, resulting in the manifestations of von Hippel-Lindau disease. VHL gene product (pVHL) loss is also found in $45-80 \%$ of sCCRCCs $(2,9,13)$. pVHL is part of the ubiquitin ligase complex, targeting $\alpha$-subunits of the heterodimeric transcription factor hypoxiainducible factor (HIF) for polyubiquitylation, and subsequent proteasomal degradation in the presence of oxygen (14). pVHL-defective sCCRCC overproduce a variety of downstream mRNAs that are under HIF control, including vascular endothelial growth factor (VEGF), platelet-derived growth factor $\mathrm{B}$, and transforming growth factor $\alpha(2-4,9,13,14)$. Down-regulation of HIF- $\alpha$, especially the HIF- $2 \alpha$ subtype, appears necessary for renal tumour suppression by pVHL $(3,4,14)$. Up-regulation of HIF also stimulates EPO expression, albeit the putative effects of EPO specific for SCCRCC remain incompletely understood.

Cumulatively, the pVHL-HIF-VEGF relationship is responsible for the demonstrated clinical activity of VEGF antagonists in clear cell renal carcinoma, and forms a foundation for the testing of additional agents that inhibit HIF, or HIF-responsive gene products such as EPO, in this disease. In this study, the occurrence of VHL gene mutations in $43 \%$ of sCCRCC is similar to previous reports, whereas no mutations were detected in normal kidney tissues $(2,7,13,14)$. Hyper- 
Table V. Summary of HIF-1 $\alpha$, HIF-2 $\alpha$, and EPO expression in sCCRCC according to tumour stage and nuclear grade.

\begin{tabular}{|c|c|c|c|c|c|c|c|}
\hline & \multicolumn{4}{|c|}{ Stage } & \multicolumn{3}{|c|}{ Grade } \\
\hline & $\mathrm{T} 1$ & $\mathrm{~T} 2$ & $\mathrm{~T} 3$ & $\mathrm{~T} 4$ & G1 & $\mathrm{G} 2$ & G3 \\
\hline HIF- $1 \alpha^{+}$ & 33 & 12 & 7 & 1 & 14 & 29 & 10 \\
\hline HIF- $1 \alpha^{-}$ & 17 & 6 & 5 & 0 & 6 & 17 & 6 \\
\hline HIF- $2 \alpha^{+}$ & 40 & 17 & 11 & 1 & 17 & 40 & 12 \\
\hline HIF-2 $\alpha^{-}$ & 10 & 2 & 1 & 0 & 3 & 6 & 4 \\
\hline $\mathrm{EPO}^{+}$ & 45 & 16 & 11 & 0 & 15 & 42 & 15 \\
\hline $\mathrm{EPO}^{-}$ & 5 & 3 & 1 & 1 & 5 & 4 & 1 \\
\hline
\end{tabular}

methylation sites on exon 1 of the VHL gene, which causes silencing of the VHL gene, were also analyzed. No differences were found in methylation status between tumour and paired normal tissues, which is also consistent with published literature (15). The significance of similar VHL mutation frequencies in different pathological stages and nuclear grades in this study is unclear. As large patient cohorts are required to determine prognostic differences in relation to VHL mutations or downstream protein expression, it is apparent that analyses of 82 patients does not allow for pathological and prognostic correlation for sCCRCC to be determined and identifies an important area for further study as patient numbers increase. Although VHL gene status shows promise as a marker to refine prognosis and prediction of metastatic spread, initial results have proven inconsistent. VHL gene mutations and increased HIF- $1 \alpha$ and VEGF expression have been associated with aggressive-variant and advanced-stage RCC $(13,16)$. Conversely, VHL gene mutations have also been associated with improved prognosis and treatment advantage for VEGFantagonists (objective response rates and prolonged time to progression) (17). Up-regulation of HIF secondary to VHL gene inactivation plays an essential role in SCCRCC, promoting angiogenesis, the glycolytic switch, and adipose differentiation related protein (ADRP) by lipid influx, resulting in appearance of vascular neoplasms (18). Our HIF expression results confirm up-regulation of HIF- $1 \alpha$ and HIF- $2 \alpha$ proteins in sCCRCC in response to VHL gene inactivation. Our data also indicate that there is a clear difference in VHL alteration status between the HIF- $2 \alpha$ positive cases and HIF- $2 \alpha$ negative cases. The VHL gene was altered in $60.9 \%$ of the HIF- $2 \alpha$ positive cases versus $23.1 \%$ of the HIF- $2 \alpha$ negative cases. A similar relationship was observed for HIF-1 $\alpha$. However, $48.6 \%$ (18/37) and $73.0 \%$ (27/37) of sCCRCC showed expression of HIF-1a and HIF$2 \mathrm{a}$, respectively, without VHL alterations. These results are consistent with a recently reported study and may result from VHL gene inactivation occurring through non-mutational mechanisms such as gene deletion or genetic factors, causing sCCRCC sharing of the HIF pathway and downstream tumourigenesis (11). A key feature of RCC is its increased vascularity, which has been linked to the abnormal production of tumour-derived EPO driving neoangiogenesis and tumourigenesis as VHL gene function is lost $(5,10,11)$. EPO expression is also thought to correspond to microvascular invasion and aggressive of the tumour. In our study, EPO was detected in 72 sCCRCC and correlated to higher Fuhrman nuclear grade. In vitro studies suggest that EPO expression levels are regulated by $\mathrm{pVHL}$, which has been shown to control of HIF- $1 \alpha$ and HIF- $2 \alpha$ concentrations through ubiquitination and proteasomal degradation mechanisms $(19,20)$. Upregulated HIF stimulates EPO overexpression in tumours, with HIF-2 $\alpha$ more active in this context than HIF-1 $\alpha$. Our results indicate that $\mathrm{SCCRCC}$ not only share genetic and morphological features, but also a common HIF-EPO pathogenetic pathway, with VHL-associated RCCs. The expression rate of EPO in sporadic renal tumours was higher than VHL mutation rates and HIF expression. Further study of EPO biology for sCCRCC is required in this context, as findings may reflect alternative VHL gene inactivation pathways, technical limitations (for example, sensitivity of HIF antibody), or novel unknown factors governing expression.

In conclusion, hypoxia inducible factor- $1 \alpha$, hypoxia inducible factor- $2 \alpha$ and erythropoietin expression are frequently observed in sporadic clear cell renal cell carcinoma. Although both HIF forms up-regulate expression of EPO, the relationship to HIF-2 $\alpha$ appears more pronounced. Initial results are thought-provoking and identify the VHL-HIF-EPO mechanism for further study, as it may represent a new candidate of molecular target for therapy of sCCRCC.

\section{References}

1. Latif F, Tory K, Gnarra J, et al: Identification of the von HippelLindau disease tumour suppressor gene. Science 260: 1317-1320, 1993.

2. Linehan WM, Pinto PA, Srinivasan R, et al: Identification of the genes for kidney cancer: opportunity for disease-specific targeted therapeutics. Clin Cancer Res 13: 671-679, 2007.

3. Kim CM, Vocke C, Toress-Cabal C, et al: Expression of hypoxia inducible factor-1alpha and 2 alpha in genetically distinct early renal cortical tumours. J Urol 175: 1908-1914, 2006.

4. Carroll VA and Ashcroft M: Role of hypoxia-inducible factor (HIF)-1alpha versus HIF-2alpha in the regulation of HIF target genes in response to hypoxia, insulin-like growth factor-I, or loss of Hippel-Lindau function: implications for targeting the HIF pathway. Cancer Res 15: 6264-6270, 2006.

5. Lee YS, Vortmeyer AO, Lubensky IA, et al: Coexpression of erythropoietin and erythropoietin receptor in von Hippel-Lindau disease-associated renal cysts and renal cell carcinoma. Clin Cancer Res 11: 1059-1064, 2005.

6. Vogel TW, Vortmeyer AO, Lubensky IA, et al: Coexpression of erythropoietin and its receptor in endolymphatic sac tumours. J Neurosurg 103: 284-288, 2005.

7. Linehan WM, Walther MM and Zbar B: The genetic basis of cancer of the kidney. J Urol 170: 2163-2172, 2003. 
8. Gilcrease MZ, Schmidt L, Zbar B , Truong L, Rutledge M and Wheeler TM: Somatic von Hippel-Lindau mutation in clear cel papillary cystadenoma of the epididymis. Human Path 26: 1341-1346, 1995.

9. Prowse AH, Webster AR, Richards FM, et al: Somatic inactivation of the VHL gene in von Hippel-Lindau disease tumours. Am J Hum Genet 60: 765-771, 1997.

10. Hardee ME, Arcasoy MO, Blackwell KL, Kirkpatrick JP and Dewhirst MW: Erythropoietin biology in cancer. Clin Cancer Res 12: 332-339, 2006.

11. Gong K, Zhang N, Zhang Z and Na Y: Co-expression of erythopoietin and erythopoietin receptor in sporadic clear cell renal cell carcinoma. Cancer Biol Ther 5: 582-585, 2006.

12. Lam JS, Pantuck AJ, Belldegrun AS and Figlin RA: Protein expression profiles in renal cell carcinoma: staging, prognosis, and patient selection for clinical profiles. Clin Cancer Res 13: 703-708, 2007

13. Xi N, Guan W, Ryan CK, Schoen SR, Di'Santagnese PA and Messing EM: Overproduction of vascular endothelial growth factor related to von Hippel-Lindau tumour suppressor gene mutations and hypoxia-inducible factor- $1 \alpha$ expression in renal cell carcinoma. J Urol 170: 588-592, 2003.

14. Kaelin WG Jr: The von Hippel-Lindau tumour suppressor protein and clear cell renal carcinoma. Clin Cancer Res 13: 680-684, 2007.
15. Hamano K, Esumi M, Igarashi $\mathrm{H}$, et al: Biallelic inactivation of the von Hippel-Lindau tumour suppressor gene in sporadic renal cell carcinoma. J Urol 167: 713-717, 2002.

16. Brauch H, Weirich G, Brieger J, et al: VHL alterations in human clear cell renal cell carcinoma: association with advanced tumour stage and a novel hot spot mutation. Cancer Res 60: 1942-1948, 2000.

17. Rini BI, Jaeger E, Weinberg V, et al: Clinical response to therapy targeted at vascular endothelial growth factor in metastatic renal cell carcinoma: impact of patient characteristics and Von Hippel-Lindau gene status. BJU Int 98: 756-762, 2006.

18. Yao M, Huang Y, Shioi K, et al: Expression of adipose differentiation-related protein: a predictor of cancer-specific survival in clear cell renal carcinoma. Clin Cancer Res 13: 152-160, 2007.

19. Turner KJ, Moore JW, Jones A, et al: Expression of hypoxiainducible factors in human renal cancer: relationship to angiogenesis and to the von Hippel-Lindau gene mutation. Cancer Res 62: 2957-2961, 2002.

20. Ohh M, Park CW, Ivan M, et al: Ubiquitination of hypoxiainducible factor requires direct binding to the beta-domain of the von Hippel-Lindau protein. Nat Cell Biol 2: 423-426, 2002. 